\title{
DESENVOLVIMENTO DE NORMAS DRIS E CND E AVALIAÇÃO DO ESTADO NUTRICIONAL DA CULTURA DO ALGODOEIRO $^{(1)}$
}

\author{
Ademar Pereira Serra ${ }^{(2)}$, Marlene Estevão Marchetti ${ }^{(3)}$, Antonio Carlos Tadeu \\ Vitorino $^{(3)}$, José Oscar Novelino ${ }^{(3)} \&$ Marcos Antonio Camacho ${ }^{(4)}$
}

\begin{abstract}
RESUMO
Entre os vários métodos de avaliação do estado nutricional das plantas, encontram-se o DRIS e o CND, os quais têm como objetivo, entre outros, ordenar a limitação dos nutrientes na planta. Este trabalho teve como finalidade o desenvolvimento de normas DRIS e CND e a comparação dos diagnósticos realizados por esses métodos, ao avaliar lavouras comerciais de algodão, no município de São Desidério (BA). A base de dados foi constituída pelos teores totais de nutrientes de 65 amostras de folha completa e pela produtividade de algodão em caroço oriundo de talhões com média de 120 ha. Para ajuste do método, a população foi dividida em duas classes: uma com produtividade acima de $4.250 \mathrm{~kg} \mathrm{ha}^{-1} \mathrm{e}$ outra com produtividade abaixo desta. As melhores respostas em termos de produtividade foram proporcionadas pelo $\mathrm{K}$, Ca e $\mathrm{Mn}$, tanto pelo método DRIS quanto pelo CND. Avaliando a frequência de diagnoses concordantes quanto ao potencial de resposta positivo à adubação, constatou-se que o $\mathrm{N}(70,8 \%)$ foi o nutriente em que se observou a menor concordância entre os dois métodos (DRIS e CND), no entanto, o método DRIS diagnosticou maior deficiência relativa para o N do que o CND. Quanto aos demais nutrientes avaliados, a frequência de diagnoses concordantes pelo potencial de resposta à adubação esteve em torno de $73,8 \%$ para o $\mathrm{Zn}$ a 87,7 \% para o $\mathrm{P}$. Com o ajuste dos métodos DRIS e CND, chegou-se a diagnósticos semelhantes em relação ao estado nutricional da cultura do algodoeiro no oeste da Bahia.
\end{abstract}

Termos de indexação: nutrição mineral, Gossypium hirsutum, diagnose foliar, equilíbrio nutricional.

(1) Artigo extraído da Tese de Mestrado apresentada à Universidade Federal da Grande Dourados - UFGD. Trabalho financiado pelo CNPq. Recebido para publicação em março de 2009 e aprovado em outubro de 2009.

(2) Doutorando em Produção Vegetal, Universidade Federal da Grande Dourados - UFGD. Rod MS 270, km 12, Caixa Postal 533, CEP 79804-970 Dourados (MS). E-mail: ademar.serra@hotmail.com.

${ }^{(3)}$ Professor(a) da Faculdade de Ciências Agrárias, UFGD. E-mails: marlenemarchetti@ufgd.edu.br; antoniovitorino@ufgd.edu.br; josenovelino@ufgd.edu.br

${ }^{(4)}$ Professor da Universidade Estadual de Mato Grosso do Sul - UEMS. Rod. Aquidauana/Piraputanga, km 12, Caixa postal 25, CEP 79200-000 Aquidauana (MS). E-mail: camacho@uems.br 


\title{
SUMMARY: DEVELOPMENT OF DRIS AND CND NORMS AND EVALUATION OF NUTRITIONAL STATUS OF COTTON
}

\begin{abstract}
Among the different methods of assessing the nutritional status of plants, DRIS and CND have the main purpose of ranking the limiting nutrients. This study aimed to develop DRIS and CND norms and compare the diagnoses of these methods in evaluations of cotton cash crops in the region of São Desidério, state of Bahia, Brazil. The database consisted of the total nutrient content of 65 whole leaf samples and seed cotton yield of plots with an average size of 120 ha. To adjust the method the population was divided into two production classes, one with yield above and the other below 4,250 $\mathrm{kg} \mathrm{ha}^{-1}$. The best response in terms of yield was observed for potassium, calcium and manganese by DRIS as well as CND. In the assessment of the frequency of concordant diagnoses for the potential of positive response to fertilization, it was found that $N(70.8 \%)$ was the nutrient with best agreement between the two methods (DRIS and CND) whereas DRIS diagnosed greater relative deficiency for N than CND. For the other nutrients, the frequency of concordant diagnoses based on the potential response to fertilization was from around $73.8 \%$ for Zn to $87.7 \%$ for P. With the adjustment of the methods DRIS and $C N D$, the diagnoses of the nutritional status of cotton in western Bahia were similar.
\end{abstract}

Index terms: Mineral nutrition, Gossypium hirsutum, leaf analysis, nutritional balance.

\section{INTRODUÇÃO}

O conhecimento do equilíbrio nutricional das plantas é importante para que se possa avaliar o potencial de rendimento e buscar o equilíbrio nutricional. A avaliação do estado nutricional de plantas por meio dos métodos de diagnose foliar, os quais relacionam os teores de nutrientes entre si, como no caso do Sistema Integrado de Diagnose e Recomendação (DRIS) e Diagnose da Composição Nutricional (CND), vem se destacando dos tradicionais, que consideram os teores dos nutrientes individualmente, como o nível crítico e a faixa de suficiência.

Nessa nova tendência, destaca-se o DRIS, proposto por Beaufils (1973), que é o principal método bivariado utilizado. Foi desenvolvido com o objetivo de classificar os nutrientes quanto à ordem de limitação ao crescimento e desenvolvimento das plantas, independentemente da idade ou do órgão da planta a ser amostrada. A partir do DRIS, são calculados índices que expressam o equilíbrio relativo dos nutrientes numa planta, por meio da comparação de relações duais (N/P, P/K, K/Ca, Ca/Mg, etc.) na amostra a ser diagnosticada, com valores-padrão ou normas.

Além do DRIS, tem-se a Diagnose da Composição Nutricional (CND), sendo esse um método que relaciona os teores dos nutrientes de forma multivariada, desenvolvido por Parent \& Dafir (1992) com base no método de análise composicional de Aitchison (1982), concernente à análise estatística de dados de composição, baseando-se na obtenção de variáveis multinutrientes $(V i)$, cada uma delas ponderada pela média geométrica da composição nutricional.
Enquanto o emprego do método DRIS pressupõe que as relações entre os teores de dois nutrientes (relações bivariáveis) sejam as melhores indicadoras do desequilíbrio nutricional (Wadt, 1996), as relações entre o teor de um nutriente e a média geométrica dos teores dos demais componentes da matéria secainclusive aqueles não avaliados analiticamente (relações multivariáveis) - são consideradas como forma de expressão do equilíbrio nutricional, quando se emprega o método CND (Parent \& Dafir, 1992).

Estudos em que se utiliza a diagnose foliar têm sido eficientes, pois a planta é o próprio extrator de nutrientes do solo, o que possibilita um diagnóstico nutricional direto (Beaufils, 1973). A correta interpretação de resultados de análises foliares proporciona informações que favorecem o uso racional de insumos, evita desperdício, melhora o equilíbrio nutricional das plantas e, consequentemente, proporciona aumento da produtividade. Portanto, preconiza-se a utilização de métodos que disponibilizem subsídios para um diagnóstico nutricional eficiente e prático, a partir de resultados analíticos das folhas de uma planta e, ou, lavoura (Partelli et al., 2005).

Segundo Urano et al. (2006), a compreensão dos princípios considerados por diferentes métodos de diagnose, bem como a comparação de seus resultados, é importante para utilização criteriosa dessas ferramentas de análise.

Este trabalho teve como objetivo o desenvolvimento de normas DRIS e CND e a comparação dos diagnósticos realizados por esses métodos, ao avaliar o estado nutricional de lavouras comerciais de algodão na região oeste do Estado da Bahia. 


\section{MATERIAL E MÉTODOS}

Este trabalho foi realizado em lavouras comerciais de algodão, no ano agrícola de 2004/2005, no município de São Desidério (BA), coordenadas geográficas $12^{\circ} 41^{\prime} \mathrm{S}$ e $45^{\circ} 40^{\prime} \mathrm{W}$, com uma altitude média de $497 \mathrm{~m}$. O solo da região de estudo é um Latossolo Amarelo distrófico psamítico, franco-argiloarenoso. A precipitação média anual encontra-se em torno de $1.700 \mathrm{~mm}$. As lavouras foram conduzidas em um sistema de plantio convencional em sucessão com a cultura da soja. As variedades que compuseram a base de dados foram: Delta Opal, Delta Penta e Acala 90.

Foram utilizadas para compor a base de dados 65 amostras compostas de folhas completas (limbo + pecíolo); cada uma dessas amostras foi formada por 50 amostras simples, as quais foram selecionadas aleatoriamente em cada talhão comercial que apresentava uma área média de 120 ha. Coletou-se uma folha por planta, que foi retirada da quinta posição na haste principal (Malavolta, 2006), durante o período de florescimento da cultura, que compreendeu os estádios F1 a F4, segundo classificação de Marur \& Ruano (2001). As amostras foram secas em estufa com circulação forçada de ar a $65^{\circ} \mathrm{C}$, até peso constante, e passadas em moinho com peneira de $0,85 \mathrm{~mm}$ (20 mesh). A produtividade $0,85 \mathrm{~mm}$ de algodão em caroço foi avaliada após a colheita, que foi realizada com colhedora automotriz nos talhões.

Nas amostras de folhas, foram determinados os teores totais de N, P, K, Ca, Mg, S, B, Cu, Fe, Mn e Zn, conforme métodos descritos por Malavolta et al. (1997). As informações utilizadas para a formação da base de dados e para o desenvolvimento do DRIS e CND foram os teores totais de nutrientes $\left(\mathrm{mg} \mathrm{kg}^{-1}\right)$ nas folhas e a produtividade de algodão em caroço. A base de dados foi dividida em duas subpopulações: uma com produtividade acima de $4.250 \mathrm{~kg} \mathrm{ha}^{-1}$ e outra com produtividade abaixo desta.

Foi realizado teste de normalidade para a escolha dos quocientes, sendo usado o teste de Lilliefors a $5 \%$. Após a transformação, por função logarítmica neperiana, dos quocientes entre teores de nutrientes em uma amostra avaliada, os desvios desses, em relação aos valores médios dos mesmos quocientes, na população de alta produtividade, foram então determinados de acordo com Jones (1981), em unidades de desvio-padrão (s), utilizando-se um fator de ajuste (c) $=1$, conforme sugerido por Wadt et al. (1998):

$$
Z\left(\frac{A}{B}\right)=\left[\left(\frac{A}{B}\right)-\left(\frac{a}{b}\right)\right] \cdot\left(\frac{c}{s}\right)
$$

Os índices DRIS para cada nutriente consistiram da média aritmética das relações diretas $(A / B)$ e inversas $(B / A)$, transformadas em variáveis normais reduzidas, de acordo com Alvarez V. \& Leite (1999), em que $n$ é o número de nutrientes envolvidos na análise:

$$
I A=\frac{\left[Z\left(\frac{A}{B}\right)+Z\left(\frac{A}{C}\right)+Z\left(\frac{A}{N}\right)+\ldots-Z\left(\frac{B}{A}\right)-Z\left(\frac{N}{A}\right)\right]}{2(n-1)}
$$

As variáveis multinutrientes $(V A)$ consistiram do logaritmo neperiano do quociente entre a concentração de cada nutriente $(A)\left(\mathrm{mg} \mathrm{kg}^{-1}\right)$ e a média geométrica das concentrações dos constituintes da massa da matéria seca $(G)$, adaptado de Khiari et al. (2001):

$$
G=(A x B x \ldots x C x R)^{1 /(\mathrm{n}+1)}
$$

Os índices CND (IA) foram calculados pela diferença entre as variáveis multinutrientes, no talhão avaliado $(V A)$ e na média da população de referência $\left(V A^{*}\right)$, dividido pelo desvio-padrão desta variável, na população de referência $\left(S A^{*}\right)$ :

$$
V A=\ln \left(\frac{A}{G}\right)
$$

O somatório dos valores em módulo dos índices DRIS ou CND, de todos os nutrientes, constituiu o índice de equilíbrio nutricional (IEN) da amostra sob diagnose:

$$
I A=\frac{V A-V A^{*}}{S A^{*}}
$$

$\mathrm{O}$ índice de equilíbrio nutricional médio (IENm) foi obtido dividindo-se o valor do índice de equilíbrio nutricional (IEN) pelo número de nutrientes avaliados (n) (Wadt, 1996):

$$
I E N m=(|I A|+|I B|+|I C|+\ldots+|I N|) / \mathrm{n}
$$

A interpretação dos índices DRIS e CND foi realizada tendo-se classificado os nutrientes de acordo com o potencial de resposta à adubação (PRA) (Wadt, 1996). Para maior síntese, as classes de potencial de resposta positiva (p) e positiva ou nula (pz) e, também, negativa (n) e negativa ou nula (nz) foram agrupadas e denominadas de limitantes por falta (LF) ou por excesso (LE), e a classe de resposta nula (z) foi denominada de não limitante (NL), conforme Silva (2001).

Para os métodos DRIS e CND, verificou-se a hipótese de que a frequência com que cada nutriente ocorreu como o limitante primário por deficiência, ou seja, com potencial de resposta à adubação positiva e de alta probabilidade, tenha sido atribuída ao acaso. Para isso, utilizou-se o teste de qui-quadrado de ajustamento (Silva et al., 2005), a $5 \%$ de probabilidade, com n-1 graus de liberdade ( $\mathrm{n}=$ número de nutrientes analisados).

Como limitante primário por deficiência considerou-se o nutriente que apresentou o índice negativo, de menor valor em relação aos índices dos demais nutrientes, e maior em módulo que o índice de equilíbrio nutricional médio $(I E N m)$. Se verdadeira a hipótese considerada, as frequências observadas para todos os nutrientes seriam estatisticamente iguais entre si (Urano et al., 2006). As frequências esperadas $(F E)$ e observadas $(F O)$ foram calculadas da seguinte forma:

$$
\begin{gathered}
F E(\%)=\left[\left(\frac{n^{0} \text { total de talhões avaliados }}{n^{0} \text { de nutrientes avaliados }}\right) /\left(n^{0} \text { total de talhões avaliados }\right)\right] .100 \\
F O(\%)=\left(\frac{n^{0} \text { de talhões em que o nutriente foi }(p)}{n^{0} \text { total de talhões avaliados }}\right) \cdot 100
\end{gathered}
$$




\section{RESULTADOS E DISCUSSÃO}

Das 65 amostras, $40(61,5 \%)$ apresentaram produtividade acima da média $\left(4.250 \mathrm{~kg} \mathrm{ha}^{-1}\right)$, formando assim a subpopulação de alta produtividade. Os 25 talhões restantes $(38,5 \%)$ constituíram, portanto, a subpopulação de baixa produtividade.

As relações duais entre os nutrientes foram transformadas usando logaritmo neperiano, favorecendo assim a aceitação da hipótese de normalidade da distribuição de frequência para $95,5 \%$ dos quocientes entre teores de nutrientes na subpopulação de alta produtividade. Essa transformação logarítmica é sugerida por Beverly (1987) como forma de correção dos desvios de simetria dos valores dos quocientes.

Urano et al. (2006) encontraram uma aceitação de 58,2 \% das relações duais sem transformação; após a transformação por logaritmo neperiano, houve aceitação de $94,5 \%$ dos quocientes da subpopulação de alta produtividade, sendo uma condição desejada para a correta utilização do método DRIS. Essa transformação é sugerida por Beverly (1987), a qual possibilita a utilização de todas as relações duais entre os nutrientes. Dessa forma, os valores das relações duais que foram submetidos à transformação usando logaritmo neperiano formaram as normas DRIS (média e desvio-padrão) do presente trabalho (Quadro 1).

Quadro 1. Médias e desvio-padrão (s) dos quocientes entre teores de nutrientes em folhas de algodão, na subpopulação de alta produtividade ${ }^{(1)}$, transformados por função logarítmica neperiana (normas DRIS), em amostras coletadas na região oeste do Estado da Bahia, no ano agrícola de 2004/2005

\begin{tabular}{|c|c|c|c|c|c|c|c|}
\hline Variável & Média & $\mathbf{s}$ & $\mathbf{p}^{(2)}$ & Variável & Média & $\mathbf{s}$ & $\mathbf{p}^{(2)}$ \\
\hline $\mathrm{N} / \mathrm{P}$ & 2,6832 & 0,1713 & $>0,05$ & $\mathrm{~S} / \mathrm{Fe}$ & 2,9964 & 0,2727 & $>0,05$ \\
\hline $\mathrm{N} / \mathrm{K}$ & 0,7077 & 0,2559 & $>0,05$ & $\mathrm{~S} / \mathrm{Zn}$ & 3,8005 & 0,2715 & $>0,05$ \\
\hline $\mathrm{N} / \mathrm{Ca}$ & 0,4960 & 0,1630 & $>0,05$ & $\mathrm{~S} / \mathrm{Cu}$ & 5,9301 & 0,2601 & $>0,05$ \\
\hline $\mathrm{N} / \mathrm{Mg}$ & 1,6651 & 0,1957 & $>0,05$ & $\mathrm{~S} / \mathrm{Mn}$ & 4,5257 & 0,3546 & $>0,05$ \\
\hline N/S & 2,2327 & 0,2832 & $>0,05$ & $\mathrm{~S} / \mathrm{B}$ & 4,7266 & 0,2760 & $>0,05$ \\
\hline $\mathrm{N} / \mathrm{Fe}$ & 5,2291 & 0,0715 & $>0,05$ & $\mathrm{Fe} / \mathrm{N}$ & $-5,2291$ & 0,0715 & $>0,05$ \\
\hline $\mathrm{N} / \mathrm{Zn}$ & 6,0332 & 0,0706 & $>0,05$ & $\mathrm{Fe} / \mathrm{P}$ & $-2,5459$ & 0,1366 & $>0,05$ \\
\hline $\mathrm{N} / \mathrm{Cu}$ & 8,1628 & 0,1219 & $>0,05$ & $\mathrm{Fe} / \mathrm{K}$ & $-4,5213$ & 0,2331 & $>0,05$ \\
\hline $\mathrm{N} / \mathrm{Mn}$ & 6,7584 & 0,1353 & $>0,05$ & $\mathrm{Fe} / \mathrm{Ca}$ & $-4,7331$ & 0,1789 & $>0,05$ \\
\hline N/B & 6,9593 & 0,0714 & $>0,05$ & $\mathrm{Fe} / \mathrm{Mg}$ & $-3,5640$ & 0,1798 & $>0,05$ \\
\hline $\mathrm{P} / \mathrm{N}$ & $-2,6832$ & 0,1713 & $>0,05$ & $\mathrm{Fe} / \mathrm{S}$ & $-2,9964$ & 0,2727 & $>0,05$ \\
\hline $\mathrm{P} / \mathrm{K}$ & $-1,9755$ & 0,2588 & $>0,05$ & $\mathrm{Fe} / \mathrm{Zn}$ & 0,8041 & 0,0216 & $>0,05$ \\
\hline $\mathrm{P} / \mathrm{Ca}$ & $-2,1872$ & 0,2476 & $>0,05$ & $\mathrm{Fe} / \mathrm{Cu}$ & 2,9337 & 0,1068 & $>0,05$ \\
\hline $\mathrm{P} / \mathrm{Mg}$ & $-1,0181$ & 0,2455 & $>0,05$ & $\mathrm{Fe} / \mathrm{Mn}$ & 1,5293 & 0,1704 & $<0,05$ \\
\hline $\mathrm{P} / \mathrm{S}$ & $-0,4505$ & 0,2787 & $>0,05$ & $\mathrm{Fe} / \mathrm{B}$ & 1,7303 & 0,0451 & $>0,05$ \\
\hline $\mathrm{P} / \mathrm{Fe}$ & 2,5459 & 0,1366 & $>0,05$ & $\mathrm{Zn} / \mathrm{N}$ & $-6,0332$ & 0,0706 & $>0,05$ \\
\hline $\mathrm{P} / \mathrm{Zn}$ & 3,3499 & 0,1418 & $>0,05$ & $\mathrm{Zn} / \mathrm{P}$ & $-3,3499$ & 0,1418 & $>0,05$ \\
\hline $\mathrm{P} / \mathrm{Cu}$ & 5,4795 & 0,1743 & $>0,05$ & $\mathrm{Zn} / \mathrm{K}$ & $-5,3254$ & 0,2337 & $>0,05$ \\
\hline $\mathrm{P} / \mathrm{Mn}$ & 4,0752 & 0,2386 & $>0,05$ & $\mathrm{Zn} / \mathrm{Ca}$ & $-5,5371$ & 0,1740 & $>0,05$ \\
\hline $\mathrm{P} / \mathrm{B}$ & 4,2761 & 0,1468 & $>0,05$ & $\mathrm{Zn} / \mathrm{Mg}$ & $-4,3681$ & 0,1767 & $>0,05$ \\
\hline $\mathrm{K} / \mathrm{N}$ & $-0,7077$ & 0,2559 & $>0,05$ & $\mathrm{Zn} / \mathrm{S}$ & $-3,8005$ & 0,2715 & $>0,05$ \\
\hline $\mathrm{K} / \mathrm{P}$ & 1,9755 & 0,2588 & $>0,05$ & $\mathrm{Zn} / \mathrm{Fe}$ & $-0,8041$ & 0,0216 & $>0,05$ \\
\hline $\mathrm{K} / \mathrm{Ca}$ & $-0,2117$ & 0,2863 & $<0,05$ & $\mathrm{Zn} / \mathrm{Cu}$ & 2,1296 & 0,0980 & $>0,05$ \\
\hline $\mathrm{K} / \mathrm{Mg}$ & 0,9573 & 0,3280 & $>0,05$ & $\mathrm{Zn} / \mathrm{Mn}$ & 0,7252 & 0,1707 & $>0,05$ \\
\hline $\mathrm{K} / \mathrm{S}$ & 1,5250 & 0,3955 & $>0,05$ & $\mathrm{Zn} / \mathrm{B}$ & 0,9262 & 0,0414 & $>0,05$ \\
\hline $\mathrm{K} / \mathrm{Fe}$ & 4,5213 & 0,2331 & $>0,05$ & $\mathrm{Cu} / \mathrm{N}$ & $-8,1628$ & 0,1219 & $>0,05$ \\
\hline $\mathrm{K} / \mathrm{Zn}$ & 5,3254 & 0,2337 & $>0,05$ & $\mathrm{Cu} / \mathrm{P}$ & $-5,4795$ & 0,1743 & $>0,05$ \\
\hline $\mathrm{K} / \mathrm{Cu}$ & 7,4550 & 0,2387 & $>0,05$ & $\mathrm{Cu} / \mathrm{K}$ & $-7,4550$ & 0,2387 & $>0,05$ \\
\hline $\mathrm{K} / \mathrm{Mg}$ & 6,0506 & 0,3008 & $>0,05$ & $\mathrm{Cu} / \mathrm{Ca}$ & $-7,6667$ & 0,1583 & $>0,05$ \\
\hline $\mathrm{K} / \mathrm{B}$ & 6,2516 & 0,2409 & $>0,05$ & $\mathrm{Cu} / \mathrm{Mg}$ & $-6,4977$ & 0,2108 & $>0,05$ \\
\hline $\mathrm{Ca} / \mathrm{N}$ & $-0,4960$ & 0,1630 & $>0,05$ & $\mathrm{Cu} / \mathrm{S}^{\circ}$ & $-5,9301$ & 0,2601 & $>0,05$ \\
\hline $\mathrm{Ca} / \mathrm{P}$ & 2,1872 & 0,2476 & $>0,05$ & $\mathrm{Cu} / \mathrm{Fe}$ & $-2,9337$ & 0,1068 & $<0,05$ \\
\hline $\mathrm{Ca} / \mathrm{K}$ & 0,2117 & 0,2863 & $<0,05$ & $\mathrm{Cu} / \mathrm{Zn}$ & $-2,1296$ & 0,0980 & $>0,05$ \\
\hline $\mathrm{Ca} / \mathrm{Mg}$ & 1,1691 & 0,2429 & $>0,05$ & $\mathrm{Cu} / \mathrm{Mn}$ & $-1,4044$ & 0,1966 & $>0,05$ \\
\hline $\mathrm{Ca} / \mathrm{S}$ & 1,7367 & 0,3296 & $>0,05$ & $\mathrm{Cu} / \mathrm{B}$ & $-1,2034$ & 0,0972 & $>0,05$ \\
\hline $\mathrm{Ca} / \mathrm{Fe}$ & 4,7331 & 0,1789 & $>0,05$ & $\mathrm{Mn} / \mathrm{N}$ & $-6,7584$ & 0,1353 & $>0,05$ \\
\hline $\mathrm{Ca} / \mathrm{Zn}$ & 5,5371 & 0,1740 & $>0,05$ & $\mathrm{Mn} / \mathrm{P}$ & $-4,0752$ & 0,2386 & $>0,05$ \\
\hline $\mathrm{Ca} / \mathrm{Cu}$ & 7,6667 & 0,1583 & $>0,05$ & $\mathrm{Mn} / \mathrm{K}$ & $-6,0506$ & 0,3008 & $>0,05$ \\
\hline $\mathrm{Ca} / \mathrm{Mn}$ & 6,2624 & 0,2109 & $>0,05$ & $\mathrm{Mn} / \mathrm{Ca}$ & $-6,2624$ & 0,2109 & $>0,05$ \\
\hline $\mathrm{Ca} / \mathrm{B}$ & 6,4633 & 0,1657 & $>0,05$ & $\mathrm{Mn} / \mathrm{Mg}$ & $-5,0933$ & 0,2560 & $>0,05$ \\
\hline $\mathrm{Mg} / \mathrm{N}$ & $-1,6651$ & 0,1957 & $>0,05$ & $\mathrm{Mn} / \mathrm{S}$ & $-4,5257$ & 0,3546 & $>0,05$ \\
\hline $\mathrm{Mg} / \mathrm{P}$ & 1,0181 & 0,2455 & $>0,05$ & $\mathrm{Mn} / \mathrm{Fe}$ & $-1,5293$ & 0,1704 & $>0,05$ \\
\hline $\mathrm{Mg} / \mathrm{K}$ & $-0,9573$ & 0,3280 & $>0,05$ & $\mathrm{Mn} / \mathrm{Zn}$ & $-0,7252$ & 0,1707 & $>0,05$ \\
\hline $\mathrm{Mg} / \mathrm{Ca}$ & $-1,1691$ & 0,2429 & $>0,05$ & $\mathrm{Mn} / \mathrm{Cu}$ & 1,4044 & 0,1966 & $>0,05$ \\
\hline $\mathrm{Mg} / \mathrm{S}$ & 0,5676 & 0,3189 & $>0,05$ & $\mathrm{Mn} / \mathrm{B}$ & 0,2010 & 0,1654 & $>0,05$ \\
\hline $\mathrm{Mg} / \mathrm{Fe}$ & 3,5640 & 0,1798 & $>0,05$ & $\mathrm{~B} / \mathrm{N}$ & $-6,9593$ & 0,0714 & $>0,05$ \\
\hline $\mathrm{Mg} / \mathrm{Zn}$ & 4,3681 & 0,1767 & $>0,05$ & $\mathrm{~B} / \mathrm{P}$ & $-4,2761$ & 0,1468 & $>0,05$ \\
\hline $\mathrm{Mg} / \mathrm{Cu}$ & 6,4977 & 0,2108 & $>0,05$ & $\mathrm{~B} / \mathrm{K}$ & $-6,2516$ & 0,2409 & $>0,05$ \\
\hline $\mathrm{Mg} / \mathrm{Mn}$ & 5,0933 & 0,2560 & $>0,05$ & $\mathrm{~B} / \mathrm{Ca}$ & $-6,4633$ & 0,1657 & $>0,05$ \\
\hline $\mathrm{Mg} / \mathrm{B}$ & 5,2943 & 0,1745 & $>0,05$ & $\mathrm{~B} / \mathrm{Mg}$ & $-5,2943$ & 0,1745 & $>0,05$ \\
\hline $\mathrm{S} / \mathrm{N}$ & $-2,2327$ & 0,2832 & $>0,05$ & $\mathrm{~B} / \mathrm{S}$ & $-4,7266$ & 0,2760 & $>0,05$ \\
\hline $\mathrm{S} / \mathrm{P}$ & 0,4505 & 0,2787 & $>0,05$ & $\mathrm{~B} / \mathrm{Fe}$ & $-1,7303$ & 0,0451 & $>0,05$ \\
\hline $\mathrm{S} / \mathrm{K}$ & $-1,5250$ & 0,3955 & $>0,05$ & $\mathrm{~B} / \mathrm{Zn}$ & $-0,9262$ & 0,0414 & $>0,05$ \\
\hline $\mathrm{S} / \mathrm{Ca}$ & $-1,7367$ & 0,3296 & $>0,05$ & $\mathrm{~B} / \mathrm{Cu}$ & 1,2034 & 0,0972 & $>0,05$ \\
\hline S/Mg & $-0,5676$ & 0,3189 & $>0,05$ & $\mathrm{~B} / \mathrm{Mn}$ & $-0,2010$ & 0,1654 & $>0,05$ \\
\hline
\end{tabular}

(1) Maior que a média, com $n=40$ talhões ${ }^{(2)}$ Probabilidade para aceitação da hipótese de normalidade de distribuição de frequências, pelo teste de Lilliefors. 
Para as variáveis multinutrientes na subpopulação de referência, houve aceitação de 100 \% à hipótese de distribuição normal de frequência, constituindo dessa forma as normas CND as médias e os desvios-padrão destas (Quadro 2). De acordo com Khiari et al. (2001), as variáveis multinutrientes são os quocientes entre o teor de um determinado nutriente e a média geométrica $(G)$ dos teores dos constituintes da matéria seca, utilizando ainda um valor de complemento $[R=$ $\left.1 \times 10^{6}-(\mathrm{A}+\mathrm{B}+\ldots+\mathrm{C})\right]$. Assim como nas normas DRIS, as variáveis multinutrientes foram transformadas por logaritmo neperiano.

Quadro 2. Média e desvio-padrão (s) das variáveis multinutrientes (V) e média geométrica dos constituintes da matéria seca (G), em folhas de algodão coletadas na região oeste do Estado da Bahia, no ano agrícola de 2004/2005, na subpopulação de alta produtividade $\mathrm{e}^{(1)}$

\begin{tabular}{lrrl}
\hline Variável & Média & $\mathbf{S}$ & $\mathbf{p}^{(2)}$ \\
\hline G & 1478,4724 & 68,3493 & na \\
VN & 3,1360 & 0,0696 & $>0,05$ \\
VP & 0,4560 & 0,1432 & $>0,05$ \\
VK & 2,4282 & 0,2256 & $>0,05$ \\
VCa & 2,6399 & 0,1554 & $>0,05$ \\
VMg & 1,4709 & 0,1769 & $>0,05$ \\
VS & 0,9033 & 0,2539 & $>0,05$ \\
VFe & $-2,0931$ & 0,0460 & $>0,05$ \\
VZn & $-2,8972$ & 0,0420 & $>0,05$ \\
VCu & $-5,0268$ & 0,0812 & $>0,05$ \\
VMn & $-3,6224$ & 0,1641 & $>0,05$ \\
VB & $-3,8234$ & 0,0464 & $>0,05$ \\
\hline
\end{tabular}

(1) Maior que a média, com $\mathrm{n}=40$ talhões. ${ }^{(2)}$ Probabilidade para aceitação da hipótese de normalidade de distribuição de frequências, pelo teste de Lilliefors; na: não avaliado.
Com o cálculo do qui-quadrado, tanto para a diagnose realizada pelo método DRIS quanto para o CND, foi possível observar que a frequência com que os nutrientes ocorreram como mais limitantes no algodoeiro não se deveu ao acaso. Assim, foi rejeitada a hipótese de que os nutrientes ocorreram como limitantes devido ao acaso e aceita a de que os métodos de diagnose DRIS e CND foram eficientes para diagnosticar diferenças de probabilidade de resposta positiva à adubação (Quadro 3). Esse resultado concorda com os obtidos por Urano et al. (2006), tanto para o DRIS quanto para o CND, na avaliação do estado nutricional da cultura da soja.

Nos talhões foi observada uma proporção de $24,62 \%$ (DRIS) e $21,54 \%$ (CND) de potencial de resposta à adubação $\mathrm{r}$ para $\mathrm{P}$. Assim, pôde-se constatar que o elemento com maior resposta negativa à adubação foi o P (Quadro 4), havendo dessa forma uma concordância entre os dois métodos, que foram capazes de diagnosticar o $\mathrm{P}$ como um dos elementos com menor proporção na resposta à adubação.

A dose de $\mathrm{P}_{2} \mathrm{O}_{5}$ aplicada nos talhões onde o $\mathrm{P}$ apresentou baixa resposta à adubação foi de $180 \mathrm{~kg} \mathrm{ha}^{-1}$ na linha de semeadura, sendo essa quantidade considerada elevada; pelo fato de esses solos apresentarem textura arenosa, a disponibilidade de P é maior que em um solo de textura argilosa, onde há maior fixação desse elemento, acarretando menor disponibilidade para as plantas. Zancanaro (2004) relata que em regiões produtoras de algodão, como no Mato Grosso, observa-se uso excessivo de $\mathrm{P}$, sem levar em consideração as análises de solo e histórico de uso, sendo comum encontrar áreas com algodão apresentando teores elevados de $\mathrm{P}$ no solo.

Os nutrientes que com maior frequência apresentaram nos talhões resposta positiva à adubação

Quadro 3. Cálculo do qui-quadrado, para as frequências observadas (\%) de N, P, K, Ca, Mg, S, B, Cu, Fe, Mn e Zn como nutriente limitante primário por deficiência ${ }^{(1)}$, pelos métodos DRIS e CND

\begin{tabular}{|c|c|c|c|c|c|c|}
\hline \multirow{2}{*}{ Nutriente } & \multicolumn{3}{|c|}{ DRIS } & \multicolumn{3}{|c|}{ CND } \\
\hline & FO & $\mathbf{F E}$ & $(\mathrm{FO}-\mathrm{FE})^{2} / \mathrm{FE}$ & FO & FE & $(\mathrm{FO}-\mathrm{FE})^{2} / \mathrm{FE}$ \\
\hline $\mathrm{N}$ & 0,000 & 9,09 & 9,09 & 1,54 & 9,09 & 6,27 \\
\hline $\mathrm{P}$ & 7,69 & 9,09 & 0,22 & 6,15 & 9,09 & 0,95 \\
\hline $\mathrm{K}$ & 23,08 & 9,09 & 21,52 & 20,00 & 9,09 & 13,09 \\
\hline $\mathrm{Ca}$ & 23,08 & 9,09 & 21,52 & 21,54 & 9,09 & 17,04 \\
\hline $\mathrm{Mg}$ & 6,15 & 9,09 & 0,95 & 3,08 & 9,09 & 3,98 \\
\hline $\mathrm{S}$ & 9,23 & 9,09 & 0,00 & 7,69 & 9,09 & 0,22 \\
\hline $\mathrm{Fe}$ & 3,08 & 9,09 & 3,98 & 6,15 & 9,09 & 0,95 \\
\hline $\mathrm{Zn}$ & 0,00 & 9,09 & 9,09 & 0,00 & 9,09 & 9,09 \\
\hline $\mathrm{Cu}$ & 7,69 & 9,09 & 0,22 & 10,77 & 9,09 & 0,31 \\
\hline $\mathrm{Mn}$ & 18,46 & 9,09 & 9,66 & 13,85 & 9,09 & 2,49 \\
\hline B & 1,54 & 9,09 & 6,27 & 6,15 & 9,09 & 0,95 \\
\hline Qui-quadrado & & & $82,508876^{* *}$ & & & $55,33728^{* *}$ \\
\hline
\end{tabular}

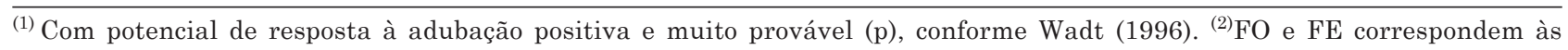
frequências observada e esperada, respectivamente. ${ }^{* *}$ : $\mathrm{p}<0,01$. 
foram K, Ca e Mn (Quadro 4). Nas avaliações em que o K e o Ca foram deficientes, os teores desses nutrientes nas análises de solo estiveram abaixo do adequado, resultando assim em deficiência desses elementos na planta, o que foi constatado pelo potencial de resposta à adubação positiva.

A resposta positiva ao $\mathrm{Mn}$ foi um resultado esperado, devido à intensidade de uso do calcário nesses talhões, chegando a doses de $7.000 \mathrm{~kg} \mathrm{ha}^{-1}$, em um solo de textura franco-arenosa, levando possivelmente à deficiência de micronutrientes, inclusive de $\mathrm{Mn}$. Carvalho et al. (2007) relatam que a deficiência de Mn tem sido constatada em solos arenosos com altas doses de calcário na Bahia, nos quais o $\mathrm{pH}$ tem atingido valores superiores a 6,3 .
Ao se avaliar a frequência de diagnoses concordantes quanto ao potencial de resposta positiva à adubação, constatou-se que o $\mathrm{N}(70,8 \%)$ foi o nutriente em que se observou a menor concordância entre os dois métodos (DRIS e CND); no entanto, o método DRIS diagnosticou maior deficiência relativa para o $\mathrm{N}$ do que o CND. Quanto aos demais nutrientes, a frequência de diagnoses concordantes pelo potencial de resposta à adubação esteve em torno de $73,8 \%$ para o $\mathrm{Zn}$ a 87,7 \% para o $\mathrm{P}$ (Quadro 5).

As cinco classes de potencial de resposta à adubação ( $p, p z, z, n z$ e n) foram agrupadas em três classes de estado nutricional (LF, NL e LE) (Quadro 6): a resposta de adubação positiva (p) e positiva ou nula (pz) foram reduzidas à limitante por falta (LF); nula (z), à não

Quadro 4. Frequência do potencial de resposta à adubação de N, P, K, Ca, Mg, S, B, Cu, Fe, Mn e Zn, determinada pelos métodos DRIS e CND, em amostras de algodão coletadas na região oeste do Estado da Bahia, no ano agrícola de 2004/2005, na subpopulação de baixa e alta produtividade

\begin{tabular}{|c|c|c|c|c|c|c|}
\hline \multirow{2}{*}{ Nutriente } & \multirow{2}{*}{ Método } & \multicolumn{5}{|c|}{ Potencial de resposta à adubação } \\
\hline & & $\mathbf{p}$ & pz & $\mathbf{z}$ & $\mathbf{n z}$ & $\mathbf{n}$ \\
\hline $\mathrm{N}$ & $\begin{array}{l}\text { DRIS } \\
\text { CND }\end{array}$ & $\begin{array}{l}0,00 \\
1,54\end{array}$ & $\begin{array}{l}16,92 \\
24,62\end{array}$ & $\begin{array}{l}-\% \\
64,62 \\
47,69\end{array}$ & $\begin{array}{r}13,85 \\
9,23\end{array}$ & $\begin{array}{r}4,62 \\
16,92\end{array}$ \\
\hline $\mathrm{P}$ & $\begin{array}{l}\text { DRIS } \\
\text { CND }\end{array}$ & $\begin{array}{l}7,69 \\
6,15\end{array}$ & $\begin{array}{r}12,31 \\
9,23\end{array}$ & $\begin{array}{l}49,23 \\
58,46\end{array}$ & $\begin{array}{l}6,15 \\
4,62\end{array}$ & $\begin{array}{l}24,62 \\
21,54\end{array}$ \\
\hline $\mathrm{K}$ & $\begin{array}{l}\text { DRIS } \\
\text { CND }\end{array}$ & $\begin{array}{l}23,08 \\
20,00\end{array}$ & $\begin{array}{r}13,85 \\
7,69\end{array}$ & $\begin{array}{l}40,00 \\
53,85\end{array}$ & $\begin{array}{l}7,69 \\
9,23\end{array}$ & $\begin{array}{r}15,38 \\
9,23\end{array}$ \\
\hline $\mathrm{Ca}$ & $\begin{array}{l}\text { DRIS } \\
\text { CND }\end{array}$ & $\begin{array}{l}23,08 \\
21,54\end{array}$ & $\begin{array}{r}10,77 \\
7,69\end{array}$ & $\begin{array}{l}50,77 \\
56,92\end{array}$ & $\begin{array}{l}9,23 \\
9,23\end{array}$ & $\begin{array}{l}6,15 \\
4,62\end{array}$ \\
\hline $\mathrm{Mg}$ & $\begin{array}{l}\text { DRIS } \\
\text { CND }\end{array}$ & $\begin{array}{l}6,15 \\
3,08\end{array}$ & $\begin{array}{l}6,15 \\
4,62\end{array}$ & $\begin{array}{l}41,54 \\
55,38\end{array}$ & $\begin{array}{l}32,31 \\
23,08\end{array}$ & $\begin{array}{l}13,85 \\
13,84\end{array}$ \\
\hline $\mathrm{S}$ & $\begin{array}{l}\text { DRIS } \\
\text { CND }\end{array}$ & $\begin{array}{l}9,23 \\
7,69\end{array}$ & $\begin{array}{l}12,31 \\
10,77\end{array}$ & $\begin{array}{l}50,77 \\
60,00\end{array}$ & $\begin{array}{l}12,31 \\
13,85\end{array}$ & $\begin{array}{r}15,38 \\
7,69\end{array}$ \\
\hline $\mathrm{Fe}$ & $\begin{array}{l}\text { DRIS } \\
\text { CND }\end{array}$ & $\begin{array}{l}3,08 \\
6,15\end{array}$ & $\begin{array}{r}4,62 \\
10,77\end{array}$ & $\begin{array}{l}86,15 \\
61,54\end{array}$ & $\begin{array}{r}1,54 \\
16,92\end{array}$ & $\begin{array}{l}4,62 \\
4,62\end{array}$ \\
\hline $\mathrm{Zn}$ & $\begin{array}{l}\text { DRIS } \\
\text { CND }\end{array}$ & $\begin{array}{l}0,00 \\
0,00\end{array}$ & $\begin{array}{l}4,62 \\
7,69\end{array}$ & $\begin{array}{l}89,23 \\
75,38\end{array}$ & $\begin{array}{r}6,15 \\
12,31\end{array}$ & $\begin{array}{l}0,00 \\
4,62\end{array}$ \\
\hline $\mathrm{Cu}$ & $\begin{array}{l}\text { DRIS } \\
\text { CND }\end{array}$ & $\begin{array}{r}7,69 \\
10,77\end{array}$ & $\begin{array}{l}18,46 \\
16,92\end{array}$ & $\begin{array}{l}61,54 \\
56,92\end{array}$ & $\begin{array}{r}9,23 \\
10,77\end{array}$ & $\begin{array}{l}3,08 \\
4,62\end{array}$ \\
\hline $\mathrm{Mn}$ & $\begin{array}{l}\text { DRIS } \\
\text { CND }\end{array}$ & $\begin{array}{l}18,46 \\
13,85\end{array}$ & $\begin{array}{l}15,38 \\
15,38\end{array}$ & $\begin{array}{l}46,15 \\
50,77\end{array}$ & $\begin{array}{r}9,23 \\
10,77\end{array}$ & $\begin{array}{r}10,77 \\
9,23\end{array}$ \\
\hline $\mathrm{B}$ & $\begin{array}{l}\text { DRIS } \\
\text { CND }\end{array}$ & $\begin{array}{l}1,54 \\
6,15\end{array}$ & $\begin{array}{l}10,77 \\
13,85\end{array}$ & $\begin{array}{l}76,92 \\
58,46\end{array}$ & $\begin{array}{r}6,15 \\
13,85\end{array}$ & $\begin{array}{l}4,62 \\
7,69\end{array}$ \\
\hline
\end{tabular}

(1) p: positiva, com alta probabilidade; pz: positiva, com baixa probabilidade; z: nula; nz: negativa, com baixa probabilidade; n: negativa, com alta probabilidade, conforme Wadt (1996).

Quadro 5. Frequência de talhões com diagnoses concordantes do potencial de resposta à adubação (PRA), determinada pelos métodos DRIS e CND, em amostras foliares de algodão coletadas na região oeste do Estado da Bahia, no ano agrícola de 2004/2005, na subpopulação de baixa e alta produtividade

\begin{tabular}{|c|c|c|c|c|c|c|c|c|c|c|c|}
\hline & $\mathbf{N}$ & $\mathbf{P}$ & $\mathbf{K}$ & $\mathbf{C a}$ & $\mathbf{M g}$ & $\mathbf{S}$ & $\mathrm{Fe}$ & $\mathrm{Zn}$ & $\mathbf{C u}$ & Mn & B \\
\hline PRA & 70,8 & 87,7 & 78,5 & 76,9 & 78,5 & 84,6 & 76,9 & 73,8 & 84,6 & 84,6 & 76,9 \\
\hline
\end{tabular}


Quadro 6. Frequência de diagnose do estado nutricional de N, P, K, Ca, Mg, S, B, Cu, Fe, Mn e Zn, determinada pelos métodos DRIS e CND, em amostras de algodão coletadas na região oeste do Estado da Bahia, no ano agrícola de 2004/2005, na subpopulação de baixa e alta produtividade

\begin{tabular}{|c|c|c|c|c|}
\hline \multirow{2}{*}{ Nutriente } & \multirow{2}{*}{ Método } & \multicolumn{3}{|c|}{ Estado nutricional } \\
\hline & & LF & NL & $\mathbf{L E}$ \\
\hline $\mathrm{N}$ & $\begin{array}{l}\text { DRIS } \\
\text { CND }\end{array}$ & $\begin{array}{l}20,00 \\
21,54\end{array}$ & $\begin{array}{c}-\% \\
58,46 \\
53,85\end{array}$ & $\begin{array}{l}21,54 \\
24,62\end{array}$ \\
\hline $\mathrm{P}$ & $\begin{array}{l}\text { DRIS } \\
\text { CND }\end{array}$ & $\begin{array}{l}18,46 \\
13,85\end{array}$ & $\begin{array}{l}50,77 \\
60,00\end{array}$ & $\begin{array}{l}30,77 \\
26,15\end{array}$ \\
\hline $\mathrm{K}$ & $\begin{array}{l}\text { DRIS } \\
\text { CND }\end{array}$ & $\begin{array}{l}36,92 \\
30,77\end{array}$ & $\begin{array}{l}40,00 \\
50,77\end{array}$ & $\begin{array}{l}23,08 \\
18,46\end{array}$ \\
\hline $\mathrm{Ca}$ & $\begin{array}{l}\text { DRIS } \\
\text { CND }\end{array}$ & $\begin{array}{l}33,85 \\
29,23\end{array}$ & $\begin{array}{l}50,77 \\
56,92\end{array}$ & $\begin{array}{l}15,38 \\
13,85\end{array}$ \\
\hline $\mathrm{Mg}$ & $\begin{array}{l}\text { DRIS } \\
\text { CND }\end{array}$ & $\begin{array}{r}12,31 \\
7,69\end{array}$ & $\begin{array}{l}41,54 \\
55,38\end{array}$ & $\begin{array}{l}46,15 \\
36,92\end{array}$ \\
\hline $\mathrm{S}$ & $\begin{array}{l}\text { DRIS } \\
\text { CND }\end{array}$ & $\begin{array}{l}20,00 \\
18,46\end{array}$ & $\begin{array}{l}53,85 \\
60,00\end{array}$ & $\begin{array}{l}26,15 \\
21,54\end{array}$ \\
\hline $\mathrm{Fe}$ & $\begin{array}{l}\text { DRIS } \\
\text { CND }\end{array}$ & $\begin{array}{r}7,69 \\
16,92\end{array}$ & $\begin{array}{l}86,15 \\
67,69\end{array}$ & $\begin{array}{r}6,15 \\
15,38\end{array}$ \\
\hline $\mathrm{Zn}$ & $\begin{array}{l}\text { DRIS } \\
\text { CND }\end{array}$ & $\begin{array}{r}4,62 \\
15,38\end{array}$ & $\begin{array}{l}84,62 \\
60,00\end{array}$ & $\begin{array}{l}10,77 \\
24,62\end{array}$ \\
\hline $\mathrm{Cu}$ & $\begin{array}{l}\text { DRIS } \\
\text { CND }\end{array}$ & $\begin{array}{l}26,15 \\
29,23\end{array}$ & $\begin{array}{l}61,54 \\
55,38\end{array}$ & $\begin{array}{l}12,31 \\
15,38\end{array}$ \\
\hline $\mathrm{Mn}$ & $\begin{array}{l}\text { DR IS } \\
\text { CND }\end{array}$ & $\begin{array}{l}33,85 \\
29,23\end{array}$ & $\begin{array}{l}44,62 \\
50,77\end{array}$ & $\begin{array}{l}21,54 \\
20,00\end{array}$ \\
\hline $\mathrm{B}$ & $\begin{array}{l}\text { DRIS } \\
\text { CND }\end{array}$ & $\begin{array}{l}12,31 \\
20,00\end{array}$ & $\begin{array}{l}76,92 \\
55,38\end{array}$ & $\begin{array}{l}10,77 \\
24,62\end{array}$ \\
\hline
\end{tabular}

LF: limitante por falta; NL: não limitante; LE: limitante por excesso, conforme Silva (2001).

Quadro 7. Frequência de talhões com diagnoses concordantes do estado nutricional (EN), determinadas pelos métodos DRIS e CND, em amostras de algodão coletadas na região oeste do Estado da Bahia, no ano agrícola de 2004/2005, na subpopulação de baixa e alta produtividade

\begin{tabular}{cccccccccccc}
\hline & $\mathbf{N}$ & $\mathbf{P}$ & $\mathbf{K}$ & $\mathbf{C a}$ & $\mathbf{M g}$ & $\mathbf{S}$ & $\mathbf{F e}$ & $\mathbf{Z n}$ & $\mathbf{C u}$ & $\mathbf{M n}$ & $\mathbf{B}$ \\
\hline \multirow{2}{*}{$\mathrm{EN}$} & 84,6 & 92,3 & 90,8 & 93,8 & 84,6 & 92,3 & 81,5 & 76,9 & 90,8 & 90,8 & 82,5 \\
\hline
\end{tabular}

limitante (NL); e negativa (n) e negativa ou nula (nz), à limitante por excesso (LE). Com esse agrupamento, observou-se que o $\mathrm{Mg}$ foi o elemento que mostrou a maior limitação por excesso: 46,16 \% (DRIS) e 36,92 \% (CND) dos talhões diagnosticados; em seguida vem o $\mathrm{P}$, com 30,77 \% (DRIS) e 26,16 \% (CND) dos talhões avaliados, aparecendo como LE.

O K, Ca e Mn foram os elementos que se apresentaram com maior frequência nos talhões como limitantes por falta, havendo concordância entre os métodos DRIS e CND (Quadro 6). Pode-se observar que, mesmo agrupando as classes de potencial de resposta à adubação para as classes do estado nutricional, houve concordância com relação aos nutrientes que tiveram uma resposta positiva (p) e negativa (n) à adubação com os limitantes por falta (LF) e limitantes por excesso (LE).

Quando se avaliou o estado nutricional, com relação à frequência dos diagnósticos concordantes, considerando os nutrientes de maneira isolada, o elemento que apresentou a menor concordância foi o $\mathrm{Zn}$, com 76,9 \% (Quadro 7). Houve maior concordância com relação aos métodos, e isso se deve à redução para três classes de potencial de resposta, pois com isso aumentou-se a probabilidade de diagnoses concordantes. 


\section{CONCLUSÕES}

1. Os métodos DRIS e CND mostraram-se igualmente satisfatórios para avaliar o estado nutricional do algodoeiro no oeste da Bahia.

2. Entre os talhões diagnosticados, o $\mathrm{Mg}$ e o $\mathrm{P}$ foram os elementos que apresentaram a maior limitação por excesso. O K, Ca e Mn foram os elementos com maior frequência nos talhões como limitantes por falta, havendo concordância entre os métodos DRIS e CND.

\section{AGRADECIMENTOS}

Ao grupo MAEDA S/A Agroindustrial, pelo apoio e pela disponibilidade dos dados, e ao $\mathrm{CNPq}$, pela bolsa de mestrado concedida.

\section{LITERATURA CITADA}

AITCHISON, J. The statistical analysis of compositional data. J. Royal Stat. Soc., 44:139-177, 1982.

ALVAREZ V., V.H. \& LEITE, R.A. Fundamentos estatísticos das fórmulas usadas para cálculo dos índices DRIS. B. Inf. Soc. Bras. Ci. Solo, 24:20-25, 1999.

BEAUFILS, E.R. Diagnosis and Recommendation Integrated System (DRIS). Pietermaritzburg, University of Natal, 1973. 132p. (Soil Sci. Bulletin, 1)

BEVERLY, R.B. Modified DRIS method for simplified nutrient diagnosis of 'Valencia' oranges. J. Plant Nutr., 10:1401. $1408,1987$.

CARVALHO, M.C.S.; FERREIRA, G.B. \& STAUT, L.B. Nutrição, calagem e adubação do algodoeiro. In: FREIRE, E.C., ed. Algodão no cerrado do Brasil. Brasília, ABRAPA, 2007. p.581-647.

JONES, C.A. Proposed modifications of the diagnosis and recommendation integrated system (DRIS) for interpreting plant analyses. Comm. Soil Sci. Plant Anal., 12:785-794, 1981.
KHIARI, L.; PARENT, L.E. \& TREMBLAY, N. Critical compositional nutrient indexes for sweet corn at early growth stage. Agron. J., 93:809-814, 2001.

MALAVOLTA, E.; VITTI, G.C. \& OLIVEIRA, S.A. Princípios, métodos e técnicas de avaliação do estado nutricional. In: MALAVOLTA, E., ed. Avaliação do estado nutricional de plantas: Princípios e aplicações. 2.ed. Piracicaba, Potafos, 1997. p.115-230.

MALAVOLTA, E. Manual de nutrição mineral de plantas. São Paulo, Agronômica Ceres, 2006. 638p.

MARUR, C.J. \& RUANO, O. A reference system for determination of developmental stages of upland cotton. R. Oleag. Fibros., 5:313-317, 2001.

PARENT, L.E. \& DAFIR, M. A theoretical concept of compositional nutrient diagnosis. J. Am. Soc. Hortic. Sci, 117:239-242, 1992.

PARTELLI, F.L.; VIEIRA, H.D. \& COSTA, A.N. Diagnóstico nutricional em cafeeiro conilon orgânico e convencional no Espírito Santo, utilizando o DRIS. Ci. Rural, 35:1456$1460,2005$.

SILVA, G.G.C. Diagnose nutricional do eucalipto pelo DRIS, M-DRIS e CND. Viçosa, MG, Universidade Federal de Viçosa, 2001. 132p. (Tese de Mestrado)

SILVA, G.G.C., NEVES, J.C.L. \& ALVAREZ V., V.H. Avaliação da universalidade das normas DRIS, M- DRIS e CND. R. Bras. Ci. Solo, 29:755-761, 2005.

URANO, E.O.M.; KURIHARA, C.H.; MAEDA, S.; VITORINO, A.C.T.; GONÇALVES, M.C. \& MARCHETTI, M.E. Avaliação do estado nutricional da soja. Pesq. Agropec. Bras., 41:1421-1428, 2006.

ZANCANARO, L. Fósforo na cultura do algodão em Mato Grosso. In: YAMADA, T \& ABDALLA, S.R.S. Fósforo na agricultura brasileira. 3.ed. Piracicaba, Potafos, 2004. p.285-289.

WADT, P.G.S. Os métodos da chance matemática e do sistema integrado de diagnose e recomendação (DRIS) na avaliação nutricional de plantios de eucalipto. Viçosa, MG, Universidade Federal de Viçosa, 1996. 123p. (Tese de Doutorado)

WADT, P.G.S.; NOVAIS, R.F.; ALVAREZ V., V.H.; FONSECA, S.; BARROS, N.F. \& DIAS, L.E. Três métodos de cálculo do DRIS para avaliar o potencial de resposta à adubação de árvores de eucalipto. R. Bras. Ci. Solo, 22:661-666, 1998. 